\title{
A Compact Design of Ultra-Wideband Antenna with Effective Triple Band-Notched Features
}

\author{
Zhou ZhengLin ${ }^{1}$, Li Ming ${ }^{2}$ \\ ${ }^{1}$ Heilongjiang Institute of Technology, Harbin 150050, China \\ ${ }^{2}$ Harbin Engineering University, Harbin 150001, China \\ ${ }^{1} 13904515640 @ 163 . c o m$
}

\begin{abstract}
A new design of ultra-wideband (UWB) antenna with triple band-notched feature is presented, in which the rectangle radiation patch is applied. The bandwidth of the UWB antenna is extended by using circle corner for the rectangle cut from the ground. A parasitic element is inserted, where an inverse U-shaped slot is cut on the top of the CPW ground plane and a U-shaped slot is cut on the rectangle radiation patch. Thus a triple band-notched feature is obtained, by which the potential interference between UWB and WLAN (Wireless Local Area Networks), C-band and X-band systems is effectively reduced. The new antenna also exhibits desirable directional radiation characteristic.
\end{abstract}

Index Terms - Ultra-Wideband Antenna, Notched Band Feature

\section{Introduction}

For past decade, the design and application of UWB communication system has been the focus of study in the field of wireless communication. As a key component for electronic information warfare, UWB antenna has attracted a lot of research interest. In 2002, the United States Federal Commission (Federal Communications Commission communication, FCC) provisions defined the UWB bandwidth for $3.1 \sim 10.6 \mathrm{GHz}$, and specified UWB antenna engineering requirements [1-2].

The operating frequency of ultra wideband communication system overlaps various narrow band systems, including wireless local area network $(5.15 \sim 5.825 \mathrm{GHz})$, satellite communication system for $\mathrm{C}$ band $(3.7 \sim 4.2 \mathrm{GHz})$ and $\mathrm{X}$ band $(7.7 \sim 8.4 \mathrm{GHz})$ etc. There exists the potential interference between the wideband and those narrow band systems. To make multiple systems compatible over these bands, a band stop filter shall be added to UWB system to eliminate the narrowband signal. Also various signal processing technologies shall be applied to improve the efficiency of spectrum utilization. However, that will result in significant increase of system complexity and design cost [3].

As known, new design of antenna with multiple stopband characteristic might be a simple and effective method to achieve ultra-wideband antenna band-notched characteristics. The most effective methods to obtain notch characteristics for antenna consist of slotting, introducing parasitic element, adding tuning unit, using factual and genetic algorithm etc. [410]. The most widely used method is slotting. As reported, two " $\mathrm{C}$ " shaped slots in the radiating patch were implemented to the circular. That creates the notch to the WLAN band and WiMAX (Worldwide Interoperability for Microwave Access) band for the antenna, and realizes the electromagnetic compatibility. Also, two "U" slots were introduced to achieve the dual band notch characteristics. Adding parasitic element is also a very common method. As reported, two different lengths of the parasitic elements were added to the monopole slot antenna, creating notch over various frequency bands.

In this paper, a new design of CPW-fed UWB antenna is presented. By introducing both parasitic elements and slots to the antenna, the notch characteristic in triple bands is realized in UWB system, in which the interference of WLAN, C band and $\mathrm{X}$ band to UWB system is eliminated, and the compatibility of the system is improved.

\section{The Antenna Structure}

The antenna is printed on medium plate with dimension of $18 \mathrm{~mm} \times 25 \mathrm{~mm} \times 1.6 \mathrm{~mm}$, its dielectric constant is 2.65 , and loss tangent angle is 0.002 , as shown in figure 1 . The antenna uses CPW-fed antenna patch on the same side of medium plate with ground. Its feed resistance is $50 \mathrm{ohm}$. The feeder line is $3.4 \mathrm{~mm}$ in width, $7.2 \mathrm{~mm}$ in length and the gap $\mathrm{g}$ to the ground is $0.3 \mathrm{~mm}$. Rectangular radiating patch is used in the new antenna design, with dimension of $10 \mathrm{~mm} \mathrm{X} 7 \mathrm{~mm}$. A rectangle groove by dimension of $16 \mathrm{mmX} 12 \mathrm{~mm}$ is opened on the floor, and the gap between $G$ radiation patch and ground is $1 \mathrm{~mm}$. The reflection coefficient of the antenna is shown in Figure 2. It is noted that the impedance bandwidth of the antenna does not meet the UWB requirement in high frequency range. For improvement, we rounded the two angles of the rectangular groove, resulting in re-distribution of the current on the floor. The impedance bandwidth of antenna at high frequencies increases as of the increase of the radius of the angle, while the impedance of medium frequency band is also influenced. Therefore $\mathrm{R}$ of $1.5 \mathrm{~mm}$ is chosen.

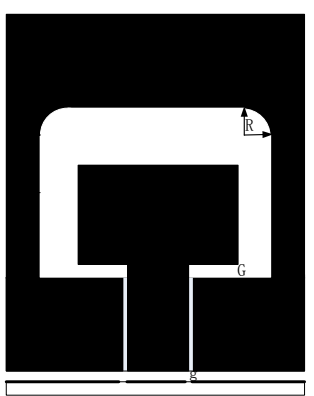

Fig.1 structure of the antenna

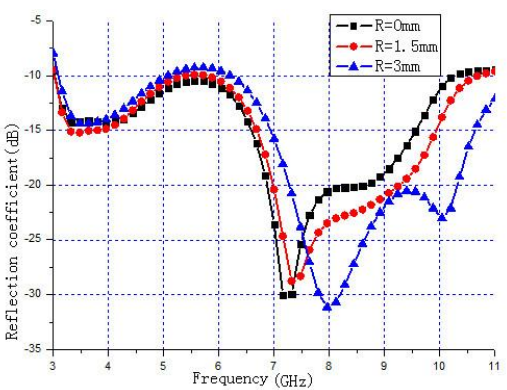

Fig. 2 reflection coefficient of the antenna 


\section{Notch Structure Analysis}

Coplanar waveguide feed is used in the antenna. The radiating patch and the floor are on the same side of dielectric plate. Notch characteristic is realized by introducing parasitic elements on the other side of the medium plate. Within the notch frequency range, the direction of the current on parasitic elements is opposite to the current direction on radiation patch. That significantly hinders the band interference for UWB system. A parasitic element with width of $0.5 \mathrm{~mm}$, where $\mathrm{L} 1=11 \mathrm{~mm}, \mathrm{~L} 2=5 \mathrm{~mm}, \mathrm{~W} 1=6.5 \mathrm{~mm}, \mathrm{~W} 2=8.7 \mathrm{~mm}$, is also introduced to the back of the antenna radiation unit, as shown in Figure 3. The effect of parasitic unit length on the notch band was analyzed by changing the length of L2, the corresponding coefficient of reflection of the antenna is shown in Figure 4. It can be seen that medium frequency moves to high frequency range when the parasitic element length was reduced. A stop band emerged for the antenna at $7.7 \sim 8.4 \mathrm{GHz}$ when $\mathrm{L} 2=5 \mathrm{~mm}$, resulting a compatible communication of UWB system and $\mathrm{X}$ band.

In addition, slotting can be also used to realize the notch characteristic. Slot can be regarded as introducing a half wavelength resonant structure. When the slot length is about the half wavelength of the medium frequency of notch band, the impedance of the antenna over the frequency and its vicinity range will become abnormal and mismatch the impedance requirement. The coefficient of reflection will increase significantly, and the notch characteristic of the antenna is exhibited over the corresponding band. The groove size can be calculated in accordance with the following formula (1) and (2). Final optimized size can be obtained by software optimizing. Notch frequency range can be adjusted with flexibility by changing the groove's length and width.

$$
\begin{aligned}
& \lambda_{n}=c /\left(f_{n} \sqrt{\varepsilon_{\text {eff }}}\right) . \\
& \mathrm{L}=\lambda_{n} / 2 .
\end{aligned}
$$

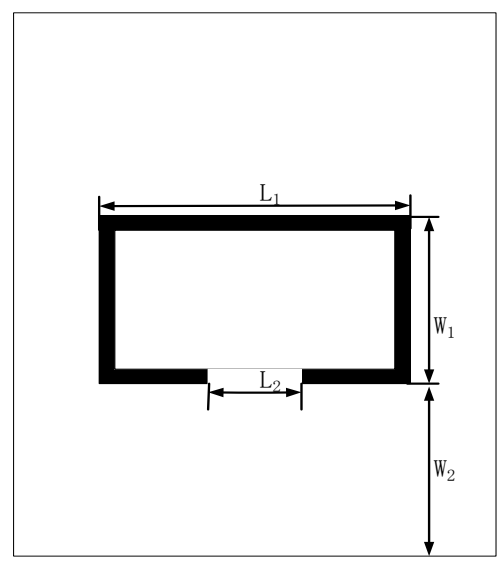

Fig. 3 parasitic element of antenna

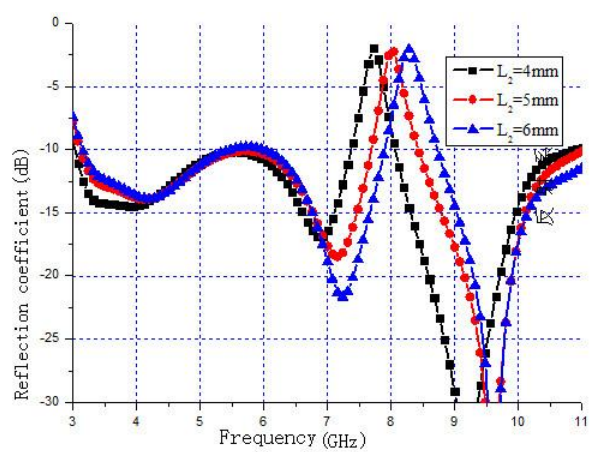

Fig. 4 coefficient of reflection with parasitic element

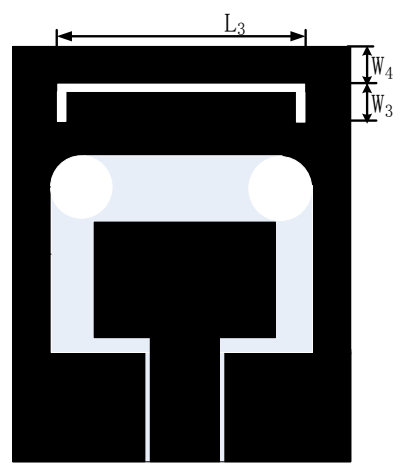

Fig. 5 Slot on the floor of the antenna

Where $\lambda_{n}$ is the medium wavelength of the notch, $C$ is the speed of light, $f_{\mathrm{n}}$ is the central frequency of the notch, $\varepsilon_{\mathrm{eff}}$ is the effective dielectric constant, $\mathrm{L}$ is the total length of groove.

Slotting can be done either on the floor of the antenna, or on the radiation unit. The sink current at corresponding wave frequency can be concentrated to the notch area by changing the antenna current distribution, while the current on both sides of notch are opposite. Thus the electric field will offset each other, and create a stop band. Firstly we make a inverted "U" shaped groove on the upper floor of the antenna, as shown in Figure 5, with dimension of width $\mathrm{W}=0.5 \mathrm{~mm}, \mathrm{~L} 3=15 \mathrm{~mm}$, $\mathrm{W} 3=2.35 \mathrm{~mm}, \mathrm{~W} 4=2.5 \mathrm{~mm}$.

The influence of the slot length on notch characteristic was analyzed. The change of coefficient of reflection with the change of slot length is displayed in Figure 6. When the slot length on upper floor is increased, the corresponding notch center will move to the low frequency range. When W3 is $2.35 \mathrm{~mm}$, the coefficient of reflection of the antenna will greater than $-10 \mathrm{~dB}$ within frequency range of $5.2 \sim 5.8 \mathrm{GHz}$, which promotes cooperative communication with WLAN. The change of coefficient of reflection of the antenna with the change of slot width is shown in Figure 7. The notch centre does not show obvious change when the slot width increases. However the width of the notch frequency band increases notably. On the other hand, the impedance characteristic of other bands is not influenced by the change of slot size. The notch band with parasitic unit is almost not affected. In order to create three notch bands, a new "U" shape slot was opened on the radiation patch, as shown in Figure 8(a), and dimension is, gap $=0.3 \mathrm{~mm}, \quad \mathrm{~L} 4=3.5 . \mathrm{mm}, \mathrm{L} 5=5.5 . \mathrm{mm}, \mathrm{W} 5=6.6 \mathrm{~mm}$, 
$\mathrm{W} 6=6.5 \mathrm{~mm}$. Thus a stop band is generated over the $3.95 \sim 4.2 \mathrm{GHz}$ range, as shown in Figure 8 (b). That inhibits the interference of the ultra-wideband antenna to $\mathrm{C}$ band communication. Figure 9 shows the surface current distribution of antenna.

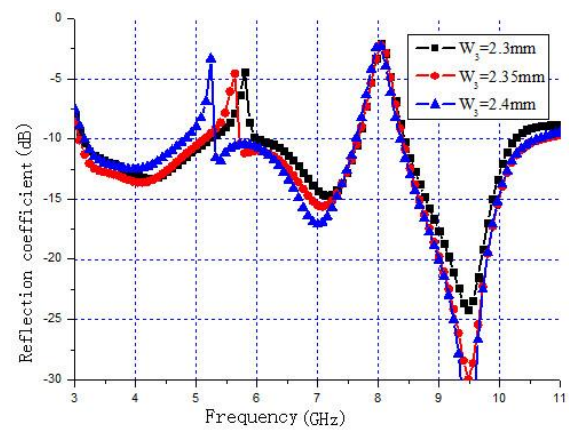

Fig.6 the change of coefficient of reflection with the change of W3

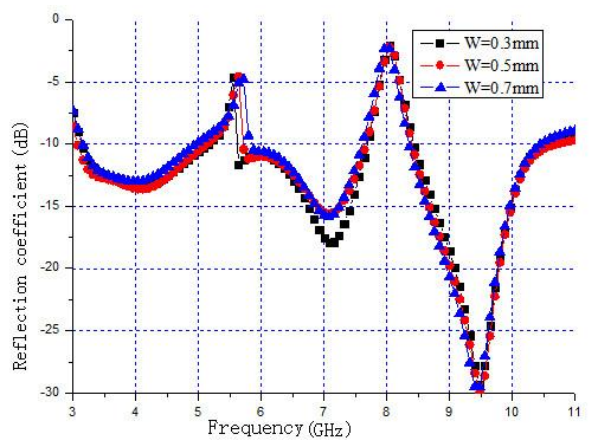

Fig. 7 the change of coefficient of reflection with the change of $\mathrm{W}$

Ultra wideband antenna requires a good directional radiation behavior. More research work has been done on radiation behavior of the antenna in frequency of $3.5 \mathrm{GHz}$, $5 \mathrm{GHz}$ and $7 \mathrm{GHz}$ respectively. A radiation simulation diagram is shown in Figure 10. Radiation of the antenna exhibits approximately omni-directional characteristics in $\mathrm{H}$ plane, and an "8" pattern in the E plane. The radiation pattern of $\mathrm{H}$ almost does not change with the increase of frequency, while there is slight distortion for radiation pattern on $\mathrm{E}$ plane. However it still meets the UWB communication requirements. In summary, the antenna possesses an excellent omni-directional radiation property.

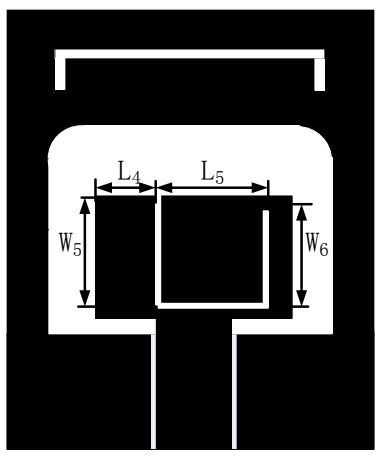

(a) "U"shape slot

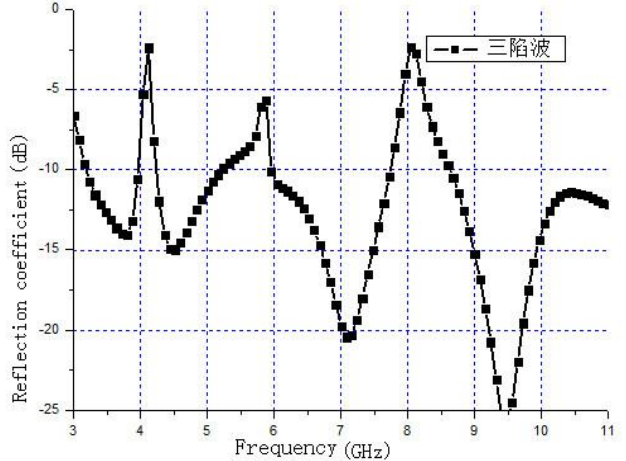

(b) coefficient of reflection

Fig.8 structure and the coefficient of reflection for antenna with triple notch characteristic

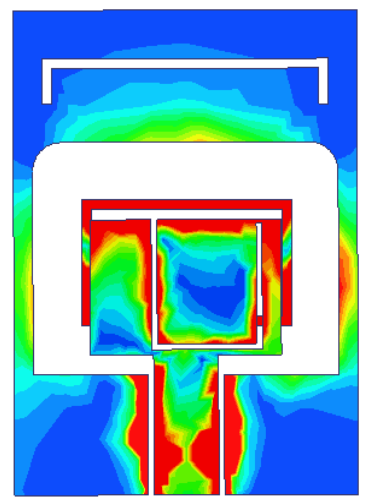

Fig. 9 surface current distribution of antenna.

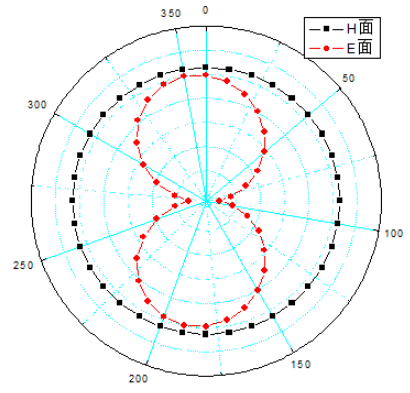

(a) $3.5 \mathrm{GHz}$

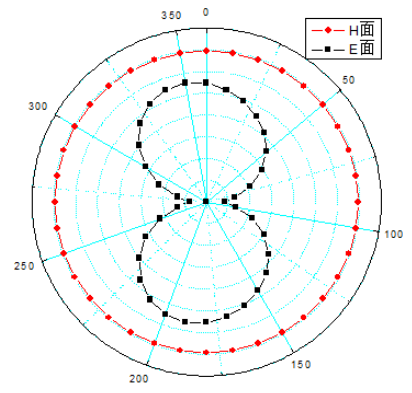

(b) $5 \mathrm{GHz}$

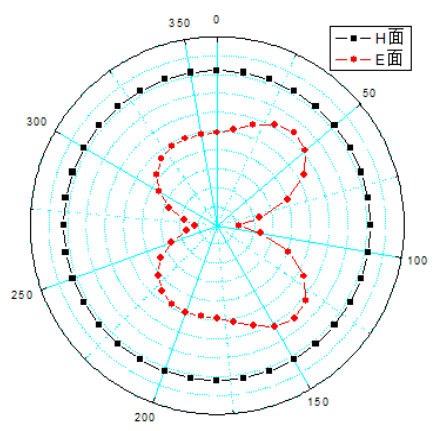

(c) $7 \mathrm{GHz}$

Fig. 10 Radiation diagram for the antenna 


\section{Conclusions}

A new design of coplanar waveguide antenna with three notch features is presented. It has compact and simple structure, while has satisfied performance and easy for integration. The rectangle radiation patch is used in the new design, and the bandwidth of the UWB antenna is extended by using circle corner for the rectangle cut from the ground. That results in antenna's reflection coefficient remaining in the range of $3.1 \mathrm{GHz}$ to $11 \mathrm{GHz}$. A parasitic element is added, whereas an inverted U-shaped slot is cut on the top of the CPW ground plane and a U-shaped slot is cut on the rectangle radiation patch. As a result, a triple band-notched characteristic is achieved, by which the potential interference between UWB and WLAN (Wireless Local Area Networks), C-band and X-band systems can be effectively reduced. Furthermore, the compatibility of the antenna is improved, and the new design antenna exhibits good radiation feature over the entire frequency range. It is believed that the new design antenna will find promising application in antenna.

\section{References}

[1] FCC 02-48, New Public Safety Applications and Broadband Internet Access among Uses Envisioned by FCC Authorization of Ultra-
Wideband Technology. Washington, D.C.: Federal Communications Commission.2002.

[2] FCC 02-48, First Report and Order in the Matter of Revision of Part 15 of the Commission's Rules Regarding Ultra-Wideband Transmission Systems. Washington, D.C., Federal Communications Commission, 2002.

[3] SCHANTZ H G, WOLENCE G, MYSZKA EM. Frequency notched UWB antennas, IEEE Conference on Ultra-Wideband Systems and Technologies, Reston VA USA, 2003: 214-218.

[4] Choi N, Jung C, Byun J, et al. Compact UWB Antenna with I-Shaped Band-Notch Parasitic Element for Laptop Applications. IEEE Antennas and Wireless Propagation Letters, 2009(8): 580-582.

[5] Razavizadeh S M R. A Band-Notched UWB Microstrip Antenna with A Resonance Back C-shaped Ring. 2010 Second International Conference on Advances in Satellite (SPA-COMM), Athens, 2010: 37-41.

[6] Z. N. Chen, Terence S.P. See, and Xianming Qing, Small printed ultrawideband antenna with reduced ground plane effect. IEEE Transactions on Antennas and Propagation, 2007:383-388.

[7] Abdollahvand M, Dadashzadeh G, Mostafa D. Compact Dual BandNotched Printed Monopole Antenna for UWB Application. IEEE Antennas and Wireless Propagation Letters, 2010(9): 1148-1151.

[8] W. J. Lui, C. H. Cheng, et al. Frequency Notched Ultra Wideband Microstrip Slot Antenna with Fractal Tuning Stub. Electronics Letters, 2005, 41(6): 294-296.

[9] W. R. Deal, V. Radisic, Yongxi Qian, T. Itoh. A broadband microstripfed slot antenna. 1999 IEEE MTT-S International Topical Symposium on Technologies for Wireless Applications, Feb. 1999, 21-24: 209-212.

[10] W. J. Lui, C. H. Cheng and H. B. Zhu. Compact Frequency Notched Ultra-Wideband Fractal Printed Slot Antenna, IEEE Microwave and Wireless Components Letters, Apr. 2006, 16(4): 224-226. 